Research Article

\title{
Response of Sesame (Sesamum indicum L.) to Sowing Methods, Nitro- gen and Sulphur Levels
}

\author{
Alam Zeb* and Amanullah Jan
}

Department of Agronomy, Faculty of Crop Production Sciences, The University of Agriculture, Peshawar, Khyber Pakbtunkbwa, Pakistan.

\begin{abstract}
Water shortage should be intimidating issue for agriculture crops. Adaptation of modern techniques and management practices in oil seed crops can reduce water shortage risks with optimum yield in oil seed crops. Sesame is being considered the most tolerant crop for avoiding drought conditions with higher yield. Experiments on "response of sesame to sowing methods, nitrogen and sulphur levels" were carried out in Agronomy Research Farms, The University of Agriculture, Peshawar. During two consecutive Kharif growing seasons of 2013 and 2014, respectively. Three sowing methods (flat, ridges and raised bed) were kept as main plot factor, whereas four nitrogen levels $\left(00,60,120\right.$, and $\left.180 \mathrm{~kg} \mathrm{ha}^{-1}\right)$ and four treatments of sulphur $\left(00,20,30\right.$ and $\left.40 \mathrm{~kg} \mathrm{ha}^{-1}\right)$, were kept as sub plot factors, which were replicated four time for each experiment. Urea and ammonium sulphate fertilizers were used as a source of nitrogen and sulfur nutrients. Results showed that all quantitative and qualitative parameters of sesame had significantly influenced by year. Study showed that raised bed sown plots significantly $(\mathrm{p} \leq 0.05)$ produced more: leaves plant ${ }^{-1}(243)$, leaves area plant ${ }^{-1}\left(444.35 \mathrm{~cm}^{2}\right)$, branchesplant $t^{-1}(9)$, plant height $(198.51 \mathrm{~cm})$, and seed yield $\left.(1339 \mathrm{~kg} \mathrm{ha})^{-1}\right)$, were recorded as compared with other sowing methods. It was also resulted from findings that $\mathrm{N}\left(180 \mathrm{~kg} \mathrm{ha}^{-1}\right)$ had significantly delayed flower and pod formation (7 and 9days) respectively as compared with control plots. Higher: leaves plant ${ }^{-1}(242)$, leaves area plant ${ }^{-1}\left(492.79 \mathrm{~cm}^{2}\right)$, branches plant ${ }^{-1}(9)$, plant height $(212.56 \mathrm{~cm})$, and seed yield $\left(1581 \mathrm{~kg} \mathrm{ha}^{-1}\right)$. Sulphur applied at $40 \mathrm{~kg} \mathrm{~S} \mathrm{ha}^{-1}$ had significantly produced more: leaves area plant ${ }^{1}\left(446.14 \mathrm{~cm}^{2}\right)$, plant height $(192.25 \mathrm{~cm})$, and seed yield $\left(1346 \mathrm{~kg} \mathrm{ha}^{-1}\right)$, as compared with lower levels of sulphur. Interactive response of $\mathrm{NxS}$ and $\mathrm{NxSxSM}$, were found significant for leaves area plant ${ }^{-1}$, plant height and seed yield. It is concluded that sowing sesame on raised bed and followed by ridges sowing method, with combined application of $180 \mathrm{~kg} \mathrm{~N} \mathrm{ha}{ }^{-1}$ and $40 \mathrm{~kg} \mathrm{~S} \mathrm{ha}^{-1}$ produced higher yield and yield related components.

Received | October 24, 2017; Accepted | December 21, 2020; Published | March 02, 2021

*Correspondence | Alam Zeb, Department of Agronomy, Faculty of Crop Production Sciences, The University of Agriculture, Peshawar, Khyber Pakhtunkhwa, Pakistan; Email: alam@aup.edu.pk

Citation $\mid$ Zeb, A. and A. Jan. 2021. Response of sesame (Sesamum indicum L.) to sowing methods, nitrogen and sulphur levels. Sarhad Journal of Agriculture, 37(1): 278-289.

DOI | http://dx.doi.org/10.17582/journal.sja/2021/37.1.278.289

Keywords | Sesame, Flat, Ridges, Raised bed, Nitrogen, Sulphur
\end{abstract}

\section{Introduction}

Sesame (Sesamum indicum L.) is an important oil S seed crop of family pedaliaceae. It is an annual short day plant, having unicullum or multi-branches, depending on variety, and gets a height 1-2 m. (Ashri,
2010) Sesame seed contains (46-64\%) oil seed content, (20 to $28 \%)$ protein, (14 to $16 \%)$ sugar and (5-7\%) minerals (Toan et al., 2010). Archeological report mentioned 2750 years ago that sesame seed were pressed manually to extract oil for different purposes, in Urartu region (SESACO, 2014). Total 
area under cultivation of sesame in Pakistan during (2014-15) was 85000 hec, with total production of 35000 tons with an average yield of $405 \mathrm{~kg} \mathrm{ha}^{-1}$. Total sesame production 0.04 (000 tonnes), with average yield of Khyber Pakhtunkhwa was recorded $1000 \mathrm{~kg}$ $\mathrm{ha}^{-1}$, which is higher than the national average yield $405 \mathrm{~kg} \mathrm{ha}^{-1}$ (DSK, 2017; MINFA, 2015-16). In 2010, Sesame seed world widely harvested about (3.84 million metric tons. Burma was the leading sesame producing country in terms of sesame seed in (201314), where India and China were leading in total production.

Sesame is being considered the most drought resistant vegetable oil crop due to its extensive root system. It has surviving adoptability features in drought climatic conditions, but in same time it also requires optimum moisture for good germination and initial seedling stands. Humidity in rainy seasons offers different fungal diseases in summer seasons (Ray, 2008). It antioxidant properties makes sesame oil the most stable, with long and better shelf life, which are considered more effective in cardio-vascular, hypercholesterolemia, hypertension, cancer diseases and immune system disorders (Kamal-Eldin et al., 2011; Cheung et al., 2007).

Sowing methods make ensure good plants establishment and later on development process. Sesame sown on ridges and raised bed has better moisture availability and retention strategies than flat sowing method (SESACO, 2014). Sesame sown on raised and ridges produced greater: capsules plant $^{-1}$, seed weight plant ${ }^{-1}$ and 1000-seed weight as compared with flat and furrow sowing methods (Malik et al., 2003). Ridge sowing method is more effective to overcome wilting, lodging, leaf spot, and stem blight diseases than flat planting pattern. Cost benefit ratio of ridge planting was higher 1:2.86, than flat sown crops. Daremcar Annual Report (20062007), mentioned that raised bed had significantly increased the cotton yield by $2.4 \% \mathrm{ha}^{-1}$ as compared with flat sowing.

Food security including edible oil production has been top on the main issue list and has been in focused of the agriculture scientist, researcher and a common man. To overcome this issue crops should produce through newly released variety with modern cropping management and inorganic management practices. Significant increase was recorded in sesame yield and yield related components with increase in nitrogen levels. Heavier seed was taken from $120 \mathrm{~kg} \mathrm{~N}$ ha $^{-1}$ (Hasanapour et al., 2012), where higher capsules and seed weight plant $^{-1}$ was increased from 0 to $80 \mathrm{~kg}$ $\mathrm{N} \mathrm{ha}^{-1}$ (Deshmukh et al., 1990). Nitrogen at $120 \mathrm{~kg}$ $\mathrm{N}$ ha ${ }^{-1}$ had enhance seed oil content (53.69\%), seed protein content $(23.46 \%)$, and oil yield (1630 kg ha ${ }^{-1}$ (Ramakrishnan et al., 1994). Yield and yield related parameters were significantlyvaried up to $150 \mathrm{~N} \mathrm{~kg} \mathrm{ha}^{-1}$ (El-Nakhlawy and Shaheen, 2009). All yield attributes were promoted except oil seed content upto $205 \mathrm{~kg}$ $\mathrm{N} \mathrm{ha}^{-1}$ (Asl, 2017; El-Nakhlawy and Shaheen, 2009).

Sulphur has a significant role in oil seed crops growth, and development and is considered the $4^{\text {th }}$ major macro nutrient for plant growth and development (Jamal et al., 2009). Sulphur plays a vital role in protein synthesis: methionine $(21 \% \mathrm{~S})$ and cysteine $(27 \% \mathrm{~S})$. Sulphur enhanced biochemical process in plant tissues which effect yield production up to $20 \mathrm{~kg}$ $\mathrm{S} \mathrm{ha}^{-1}$ (Mondal et al., 2012). Higher yield responses were recorded up to $30 \mathrm{~kg} \mathrm{~S} \mathrm{ha-1}$. Sulphur improved qualitative and quantitative parameters up to 60 $\mathrm{kg} \mathrm{S} \mathrm{ha-1} \mathrm{(Mondal} \mathrm{et} \mathrm{al.,} \mathrm{2012;} \mathrm{Raja} \mathrm{et} \mathrm{al.,} \mathrm{2007b).}$ Growth and yield of sesame enhanced with combined application of nitrogen and sulphur. Synergetic effect was found between nitrogen and sulphur nutrients (Jamal et al., 2009). $\mathrm{N}$ and $\mathrm{S}$ availability and uptake efficiency enhanced in the presence of both nitrogen and sulphur nutrients in soil (Shilpi et al., 2014). Mansoori (2012) reported that yield and oil seed content increased with application of $\mathrm{N}\left(120 \mathrm{~kg} \mathrm{ha}^{-1}\right)$ and $\mathrm{S}\left(40 \mathrm{~kg} \mathrm{~S} \mathrm{ha}^{-1}\right)$. Oil seed content increased with combined application of $60 \mathrm{~kg} \mathrm{~N} \mathrm{ha}^{-1}$ and $40 \mathrm{~kg} \mathrm{~S} \mathrm{ha}^{-1}$ (Shilpi et al., 2014).

The importance of sesame crop for seed and oil production, food industry, health concern, and economic profitability, these experiments were carried out find out the best sowing methods, nitrogen and sulphur levels for higher yield of sesame at the limited irrigated agro-climatic conditions of Peshawar-valley.

\section{Materials and Methods}

Field trails on the response of sesame to sowing methods, nitrogen and sulphur levels, were designed and completed at Agronomy Research farms (ARF), at The University of Agriculture, Peshawar- Pakistan, during kharif season on June $30^{\text {th }}$ of 2013 and 2014. Each experiment was designed in randomized 
complete block (RCB), with split plot arrangement, having three sowing methods (flat, ridge 50 and raised bed sowing methods) in main plots, three nitrogen rates $\left(60,120\right.$ and $\left.180 \mathrm{~kg} \mathrm{ha}^{-1}\right)$ and three sulphur levels $\left(20,30\right.$ and $\left.40 \mathrm{~kg} \mathrm{ha}^{-1}\right)$ were in sub main plot factor, having one control for both $\mathrm{N}$ and $\mathrm{S}$, were replicated four time. Urea (split dose $50 \%$ after $1^{\text {st }}$ irrigation on $20^{\text {th }}$ July and $50 \%$ at $2^{\text {nd }}$ irrigation on $5^{\text {th }}$ August on both years) and ammonium sulphate (whole at sowing time on $30^{\text {th }}$ June) was used as fertilizer source for $\mathrm{N}$ and $\mathrm{S}$. Each subplot size of $4 \mathrm{~m}$ $\mathrm{x} 4 \mathrm{~m}$, rows were separated by $50 \mathrm{~cm}$, and intra plant space was maintained $10 \mathrm{~cm}$ in each experiment. Variety SG-30, sown at the rate of $6 \mathrm{~kg} \mathrm{ha} \mathrm{h}^{-1}$. Being less dimension of sub plot $4 \mathrm{x} 4$, ridges $(50 \mathrm{~cm}$ ridge to ridge distance $/ 20 \mathrm{~cm}$ height $)$ and raised bed $(50 \mathrm{~cm}$ raised bed to raised bed distance $/ 20 \mathrm{~cm}$ height of the raised bed $/ 20 \mathrm{~cm}$ p-p distance) were made manually as well as with help of machinery where it was possible. Experimental soil was sandy loamy, strongly calcareous and alkaline in reaction with $\mathrm{pH}$ (8.1), EC $\left(0.28 \mathrm{dS} \mathrm{m}^{-1}\right)$, total nitrogen $(0.05 \%)$ and was marginal in AB-DTPA extractable $\mathrm{K}\left(107 \mathrm{mg} \mathrm{kg}^{-1}\right)$. Weeding, irrigation, pesticides, herbicides, activities were carried out uniformly for all the experimental units throughout the growing Kharif seasons of 2013 and 2014. Data were recorded on days to $1^{\text {st }}$ flower and pod formation, leaves, leaves area and branches plant ${ }^{-1}$, plant height $(\mathrm{cm})$, and seed yield $\left(\mathrm{kg} \mathrm{ha}^{-1}\right)$.

\section{Days to first flower and pod formation}

Data on days to first flower and capsule formation of sesame crop between flower, pod formation and date of sowing were $1^{\text {st }}$ observed and recorded in each subplot of sesame.

\section{Leaves plant ${ }^{-1}$}

Five randomly plants in each subplot were $1^{\text {st }}$ collected and leaves were counted, and its average was taken as leaves plant ${ }^{-1}$.

\section{Leaves area plant ${ }^{-1}$}

Five plantsat three different rows per plot were tagged and leaves areas were measured twice once at 35 days after sowing and then 65 days after sowing with the help of (C1-202, Portable Leaf Area Meter USA).

\section{Branches plant ${ }^{-1}$}

After harvesting ten randomly plants were collected from each sup plot of sesame and counted for branches and averaged for branches plant ${ }^{-1}$.
Plant height $(\mathrm{cm})$

Plant height $(\mathrm{cm})$ was taken in ten selected plants with help of measuring rode from top to bottom ground level.

\section{Seed yield $\left(\mathrm{kg} \mathrm{ha}^{-1}\right)$}

After threshing of four central rows in each subplot of sesame crop, seed were weighted with an electronic balance and were converted into $\mathrm{kg} \mathrm{ha}^{-1}$.

\section{Data analysis}

Different statistical tools were used to analyze and mean differences between treatments were compared for RCB design by LSD at 5\% level of probability, when the F- values was significant (Jan et al., 2009). Graphs were made with Sigma plot software (12).

\section{Results and Discussion}

\section{Days to $1^{\text {st }}$ flowering}

Flowering formation had had significantly affected by nitrogen and year (Table 1 ). As plot fertilized with 60 and $120 \mathrm{~kg} \mathrm{~N} \mathrm{ha}^{-1}$ had initiated early flowering, while late flowering was recorded in $180 \mathrm{~N} \mathrm{~kg} \mathrm{ha}$ ${ }^{1}$. Late flower formation in sesame might be due to high nitrogen and sulphur application which produce vigorous plant growth, resulted in delay flowering. Flowering delayed with increasing nitrogen and sulphur from lower to higher levels (Haruna, 2011). In year 2013, earlier flowers were produced as compared to year 2014. Delayed flowering in year 2014 might be high utilization of nutrients in the presence of nitrogen and sulphur, which prolongs vegetative growth stage. These finding are supported by (Bareja, 2011), who mentioned that soil and weather conditions can change plant growth stages (flowering stage). Significantly delay in $1^{\text {st }}$ flower formation was recorded in fertilized plots as compared with control plots. Interactions between year $(\mathrm{Y}) \mathrm{x}$ nitrogen $(\mathrm{N})$, was found significant for flower formation. Higher nitrogen application can boost vegetative growth, which resulted in late flower formation by (Raja et al., 2007).

\section{Days to $1^{\text {st }}$ pod formation}

Pod formation duration had significantly affected by nitrogen (Table 2). Year (as a factor) had also significantly influenced days to pod formation. Significantly delayed pods were produced, when sesame was fertilized with $180 \mathrm{~N} \mathrm{~kg} \mathrm{ha}{ }^{-1}$, as compared with other nitrogen levels. These changes 
in pod formation linked with different nitrogen levels application. Higher nitrogen application in sesame may boost vegetative growth with delayed pod formation process. Haruna (2011) and E1Nakhlawy and Shaheen (2009) reported that delay in pod formation was recorded with increasing level of $\mathrm{N}$ supply from 0 to $200 \mathrm{~kg} \mathrm{ha}^{-1}$. These changes in days to pod formation might be due to different weather and soil conditions of the experimental site in two consecutive years of the study. Different weather, soil moisture and nutrients bring variations in plant growth and development (Bareja, 2011). Significantly earlier $1^{\text {st }}$ pod formation $\mathrm{n}$ control plots, whereas delayed pods were recorded in fertilized plot.

Table 1: Days to $1^{\text {st }}$ flower formation of sesame as affected by sowing methods, nitrogen and sulfur levels during year 2013, and 2014.

\begin{tabular}{|c|c|c|c|}
\hline Sowing methods & 2013 & 2014 & Mean \\
\hline Ridge & 45 & 46 & 46 \\
\hline Flat bed & 45 & 46 & 46 \\
\hline Raised bed & 45 & 47 & 46 \\
\hline $\mathrm{LSD}_{(0.05)}$ & & & ns \\
\hline \multicolumn{4}{|l|}{ Nitrogen $\left(\mathrm{kg} \mathrm{ha}^{-1}\right)$} \\
\hline 60 & 44 & 46 & $45 \mathrm{~b}$ \\
\hline 120 & 44 & 46 & $45 \mathrm{~b}$ \\
\hline 180 & 46 & 48 & $47 \mathrm{a}$ \\
\hline $\mathrm{LSD}_{(0.05)}$ & & & 1 \\
\hline \multicolumn{4}{|l|}{ Sulphur $\left(\mathrm{kg} \mathrm{ha}^{-1}\right)$} \\
\hline 20 & 46 & 46 & 46 \\
\hline 30 & 45 & 47 & 46 \\
\hline 40 & 45 & 47 & 46 \\
\hline $\operatorname{LSD}_{(0.05)}$ & & & ns \\
\hline Year & 45 & 47 & $*$ \\
\hline \multicolumn{4}{|c|}{ Planned mean comparison } \\
\hline Control & 39 & 41 & 40 \\
\hline Rest & 45 & 47 & 46 \\
\hline Significance & & & $*$ \\
\hline \multicolumn{4}{|l|}{ Interactions } \\
\hline $\mathrm{Y} \times \mathrm{SM}$ & ns & $\mathrm{SM} \times \mathrm{S}$ & ns \\
\hline $\mathrm{N} \times \mathrm{S}$ & ns & $\mathrm{SM} \times \mathrm{N} \times \mathrm{S}$ & ns \\
\hline $\mathrm{Y} \times \mathrm{N}$ & $*$ & $\mathrm{Y} \times \mathrm{SM} \times \mathrm{N}$ & ns \\
\hline $\mathrm{Y} \times \mathrm{S}$ & ns & $\mathrm{Y} \times \mathrm{SM} \times \mathrm{S}$ & ns \\
\hline $\mathrm{Y} \times \mathrm{N} \times \mathrm{S}$ & ns & $\mathrm{Y} \times \mathrm{SM} \times \mathrm{N} \times \mathrm{S}$ & ns \\
\hline $\mathrm{SM} \times \mathrm{N}$ & ns & & \\
\hline
\end{tabular}

Mean followed different letters are statistically different from each at $5 \%$ of probability; *: indicate significance at $5 \%$ level of probability; ns: indicates not significant.

March 2021 | Volume 37 | Issue 1 | Page 281
Table 2: Days to $1^{\text {st }}$ pod formation of sesame as affected by sowing methods, nitrogen and sulfur levels during year 2013, and 2014.

\begin{tabular}{|c|c|c|c|}
\hline Sowing methods & 2013 & 2014 & Mean \\
\hline Ridge & 52 & 54 & 53 \\
\hline Flat bed & 52 & 54 & 53 \\
\hline Raised bed & 53 & 54 & 54 \\
\hline $\mathrm{LSD}_{(0.05)}$ & & & ns \\
\hline \multicolumn{4}{|l|}{ Nitrogen $\left(\mathrm{kg} \mathrm{ha}^{-1}\right)$} \\
\hline 60 & 52 & 54 & $53 \mathrm{~b}$ \\
\hline 120 & 52 & 54 & $53 \mathrm{~b}$ \\
\hline 180 & 55 & 56 & $55 \mathrm{a}$ \\
\hline $\operatorname{LSD}_{(0.05)}$ & & & 1 \\
\hline \multicolumn{4}{|l|}{ Sulphur $\left(\mathrm{kg} \mathrm{ha}^{-1}\right)$} \\
\hline 20 & 54 & 53 & 54 \\
\hline 30 & 53 & 54 & 54 \\
\hline 40 & 53 & 54 & 54 \\
\hline $\mathrm{LSD}_{(0.05)}$ & & & ns \\
\hline Year & 53 & 54 & $*$ \\
\hline \multicolumn{4}{|c|}{ Planned mean comparison } \\
\hline Control & 47 & 45 & 46 \\
\hline Rest & 53 & 54 & 54 \\
\hline Significance & & & $*$ \\
\hline \multicolumn{4}{|l|}{ Interactions } \\
\hline $\mathrm{Y} \times \mathrm{SM}$ & ns & $\mathrm{SM} \times \mathrm{S}$ & ns \\
\hline $\mathrm{N} \times \mathrm{S}$ & ns & $\mathrm{SM} \times \mathrm{N} \times \mathrm{S}$ & ns \\
\hline $\mathrm{Y} \times \mathrm{N}$ & $*$ & $\mathrm{Y} \times \mathrm{SM} \times \mathrm{N}$ & ns \\
\hline $\mathrm{Y} \times \mathrm{S}$ & ns & $\mathrm{Y} \times \mathrm{SM} \times \mathrm{S}$ & ns \\
\hline $\mathrm{Y} \times \mathrm{N} \times \mathrm{S}$ & ns & $\mathrm{Y} \times \mathrm{SM} \times \mathrm{N} \times \mathrm{S}$ & ns \\
\hline $\mathrm{SM} \times \mathrm{N}$ & ns & & \\
\hline
\end{tabular}

Mean followed different letters are statistically different from each at $5 \%$ of probability; *: indicate significance at $5 \%$ level of probability; ns: indicates not significant.

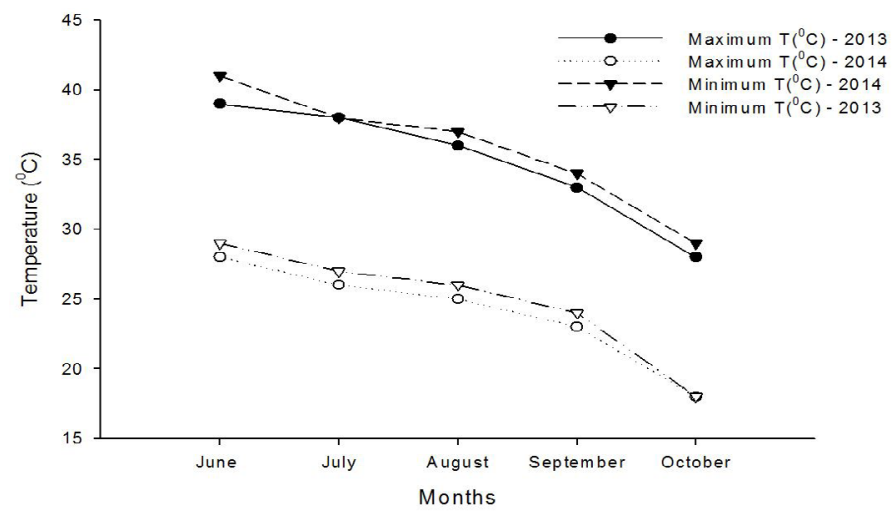

Figure 1: Monthly maximum and minimum temperature $\left({ }^{\circ} \mathrm{C}\right)$ data during sesame growing season of year 2013 and 2014 at experimental site.

Leaves plant ${ }^{-1}$

Table 3, presents data on number of leaves plant ${ }^{-1}$. 
Significantly higher number of leaves were produced by raised bed sowing method as compared with other sowing methods. Significantly more leaves plant $^{-1}$ were produced with $180 \mathrm{~kg} \mathrm{~N} \mathrm{ha}{ }^{-1}$ whereas, lower number of leaves plant ${ }^{-1}$ were produced when plots applied at $60 \mathrm{~kg} \mathrm{~N} \mathrm{ha}{ }^{-1}$. Fertilized plots had produced more leaves plant ${ }^{-1}$ as compared with control plots. Higher number leaves plant ${ }^{-1}$ were noted in year 2014. Variation in number of leaves might be due to maximum utilization of nitrogen nutrient for higher plant growth and development in different soil conditions. Leaves were increased up to $205 \mathrm{~kg} \mathrm{~N}$ $\mathrm{ha}^{-1}$ (El-Nakhlawy and Shaheen, 2009). Interaction between $\mathrm{SM} \times \mathrm{N}$, given in Figure 3, and was found significant for leaves plant ${ }^{-1}$. Nitrogen fertilization significantly encouraged leaves plant ${ }^{-1}$ in each sowing method. These variations in leaves could be considered

Table 3: Leaves plant ${ }^{-1}$ of sesame as affected by sowing methods, nitrogen and sulfur levels during year 2013 and 2014.

\begin{tabular}{|c|c|c|c|}
\hline Sowing methods & 2013 & 2014 & Mean \\
\hline Ridge & 208 & 218 & $213 \mathrm{~b}$ \\
\hline Flat & 183 & 187 & $185 \mathrm{c}$ \\
\hline Raised bed & 241 & 245 & $243 \mathrm{a}$ \\
\hline $\operatorname{LSD}_{(0.05)}$ & & & 4 \\
\hline \multicolumn{4}{|l|}{ Nitrogen $\left(\mathrm{kg} \mathrm{ha}^{-1}\right)$} \\
\hline 60 & 184 & 192 & $188 \mathrm{c}$ \\
\hline 120 & 206 & 214 & $210 \mathrm{~b}$ \\
\hline 180 & 241 & 245 & $243 \mathrm{a}$ \\
\hline $\mathrm{LSD}_{(0.05)}$ & & & 2 \\
\hline \multicolumn{4}{|l|}{ Sulphur $\left(\mathrm{kg} \mathrm{ha}^{-1}\right)$} \\
\hline 20 & 211 & 215 & 213 \\
\hline 30 & 211 & 217 & 214 \\
\hline 40 & 212 & 216 & 214 \\
\hline $\mathrm{LSD}_{(0.05)}$ & & & ns \\
\hline Year & 210 & 215 & * \\
\hline \multicolumn{4}{|c|}{ Planned mean comparison } \\
\hline Control & 133 & 143 & 138 \\
\hline Rest & 210 & 217 & 214 \\
\hline Significance & & & $*$ \\
\hline \multicolumn{4}{|l|}{ Interactions } \\
\hline $\mathrm{Y} \times \mathrm{SM}$ & ns & $\mathrm{SM} \times \mathrm{S}$ & ns \\
\hline $\mathrm{N} \times \mathrm{S}$ & ns & $\mathrm{SM} \times \mathrm{N} \times \mathrm{S}$ & ns \\
\hline $\mathrm{Y} \times \mathrm{N}$ & ns & $\mathrm{Y} \times \mathrm{SM} \times \mathrm{N}$ & $*$ \\
\hline $\mathrm{Y} \times \mathrm{S}$ & ns & $\mathrm{Y} \times \mathrm{SM} \times \mathrm{S}$ & ns \\
\hline $\mathrm{Y} \times \mathrm{N} \times \mathrm{S}$ & ns & $\mathrm{Y} \times \mathrm{SM} \times \mathrm{N} \times \mathrm{S}$ & ns \\
\hline $\mathrm{SM} \times \mathrm{N}$ & $*$ & & \\
\hline
\end{tabular}

Mean followed different letters are statistically different from each at $5 \%$ of probability; *: indicate significance at $5 \%$ level of probability; ns: indicates not significant. due to well fertile and aerated soil with optimum utilization of nutrients for plant growth. Malik et al. (2003), also reported similar results that raised bed sown plots provide more nutrients for plant growth and development.

\section{Leaves area plant ${ }^{-1}$}

Sowing methods had significantly produced higher leaf area plant ${ }^{-1}\left(444.35 \mathrm{~cm}^{2}\right)$, in raised seed bed, while lower leaves area plant ${ }^{-1}\left(428.67 \mathrm{~cm}^{2}\right)$ was recorded in flat sown plots (Table 4). Higher leaves area plant ${ }^{-1}$ were measured from plots treated with $180 \mathrm{~kg} \mathrm{~N} \mathrm{ha-}$ ${ }^{1}$, as compared with other nitrogen levels. Sulphur at $40 \mathrm{~kg} \mathrm{~S} \mathrm{ha}{ }^{-1}$ had significantly produced maximum leaves area plant ${ }^{-1}$ as compare to others sulphur levels. Control vs rest analysis of data showed that lower leaves area plant ${ }^{-1}$ was recorded in control plots, where fertilized sesame plots produced higher leaves area plant $^{-1}$. More leaves area plant ${ }^{-1}$ of sesame was recorded in year 2014, as compared with year 2013.Leaves areas difference with each factor could be considered increased in total photosynthates for maximum growth with optimum utilization of available nutrients. Higher nitrogen application increased number of leaves (Younis et al., 2020). Figure 4, shows that $(\mathrm{N})$ and $(\mathrm{S})$ combined application enhanced crop growth and development which indirectly promoted leaves parameters. Similarly, interactions between $(\mathrm{SM} \times \mathrm{N})$ Figure 5, $(\mathrm{SM} \times \mathrm{N} \times \mathrm{S})$ Figure 6, and $(\mathrm{Y}$ $\times \mathrm{SM} \times \mathrm{S})$ were found highly significant for leaves plant $^{-1}$. Figure 7 , shows that leaves area plant ${ }^{-1}$ has increased in each sowing method with increasing in nitrogen and sulphur fertilizer levels from lower to higher application. Nitrogen and sulphur application promoted leaf related parameter (Raja et al., 2007).

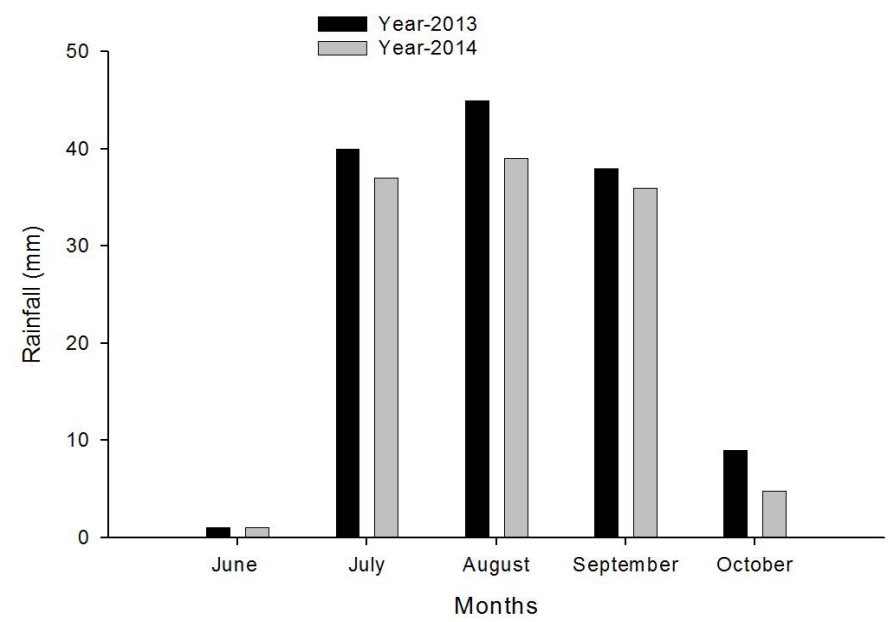

Figure 2: Monthly rainfall $(\mathrm{mm})$ data during sesame growing season of 2013 and 2014 (June to October) at experimental site. 
Table 4: Leaves area plant ${ }^{-1}\left(\mathrm{~cm}^{2}\right)$ of sesame as affected by sowing methods, nitrogen and sulfur levels during year 2013 and 2014.

\begin{tabular}{|c|c|c|c|}
\hline Sowing methods & 2013 & 2014 & Mean \\
\hline Ridge & 437.61 & 441.36 & $439.49 \mathrm{~b}$ \\
\hline Flat & 426.28 & 431.06 & $428.67 c$ \\
\hline Raised bed & 441.58 & 447.11 & $444.35 \mathrm{a}$ \\
\hline $\operatorname{LSD}_{(0.05)}$ & & & 3.0 \\
\hline \multicolumn{4}{|l|}{ Nitrogen $\left(\mathrm{kg} \mathrm{ha}^{-1}\right)$} \\
\hline 60 & 391.64 & 395.97 & $393.81 \mathrm{c}$ \\
\hline 120 & 423.44 & 428.44 & $425.90 \mathrm{~b}$ \\
\hline 180 & 490.39 & 495.19 & $492.79 \mathrm{a}$ \\
\hline $\operatorname{LSD}_{(0.05)}$ & & & 1.0 \\
\hline \multicolumn{4}{|l|}{ Sulphur $\left(\mathrm{kg} \mathrm{ha}^{-1}\right)$} \\
\hline 20 & 427.22 & 430.69 & $428.96 \mathrm{c}$ \\
\hline 30 & 434.81 & 440.00 & $437.40 \mathrm{~b}$ \\
\hline 40 & 443.44 & 448.83 & $446.14 \mathrm{a}$ \\
\hline $\mathrm{LSD}_{(0.05)}$ & & & 1.0 \\
\hline Year & 435.16 & 439.84 & * \\
\hline \multicolumn{4}{|c|}{ Planned mean comparison } \\
\hline Control & 335.42 & 340.42 & 337.92 \\
\hline Rest & 435.16 & 439.84 & 437.50 \\
\hline Significance & & & $*$ \\
\hline \multicolumn{4}{|l|}{ Interactions } \\
\hline $\mathrm{Y} \times \mathrm{SM}$ & ns & $\mathrm{SM} \times \mathrm{S}$ & ns \\
\hline $\mathrm{N} \times \mathrm{S}$ & $*$ & $\mathrm{SM} \times \mathrm{N} \times \mathrm{S}$ & $*$ \\
\hline $\mathrm{Y} \times \mathrm{N}$ & ns & $\mathrm{Y} \times \mathrm{SM} \times \mathrm{N}$ & ns \\
\hline $\mathrm{Y} \times \mathrm{S}$ & $\mathrm{ns}$ & $\mathrm{Y} \times \mathrm{SM} \times \mathrm{S}$ & $*$ \\
\hline $\mathrm{Y} \times \mathrm{N} \times \mathrm{S}$ & ns & $\mathrm{Y} \times \mathrm{SM} \times \mathrm{N} \times \mathrm{S}$ & ns \\
\hline $\mathrm{SM} \times \mathrm{N}$ & $*$ & & \\
\hline
\end{tabular}

Mean followed different letters are statistically different from each at $5 \%$ of probability; *: indicate significance at $5 \%$ level of probability; ns: indicates not significant.

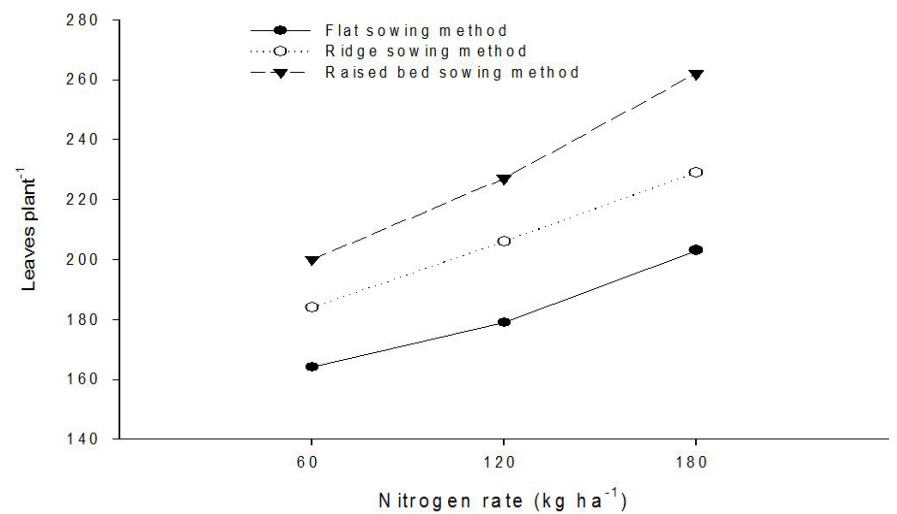

Figure 3: Interaction between (NxSM) for days to 1eave plant ${ }^{-1}$ of sesame.

\section{Branches plant ${ }^{-1}$}

Data regarding branches plant ${ }^{-1}$ are shown in Table
5. Higher number of branches plant ${ }^{-1}$ were produced in raised seed bed and ridges sown plots, respectively, while minimum branches plant ${ }^{-1}$ of were produced in flat sown plots $\mathrm{N}\left(180 \mathrm{~kg} \mathrm{ha}^{-1}\right)$ had significantly produced higher number of branches plant ${ }^{-1}$, as compared with other levels. Fertilized plots had significantly produced more number of branches plant ${ }^{-1}$, as compared to control plots. Higher branches plant ${ }^{-1}$ were recorded in $2^{\text {nd }}$ year of the study. Well

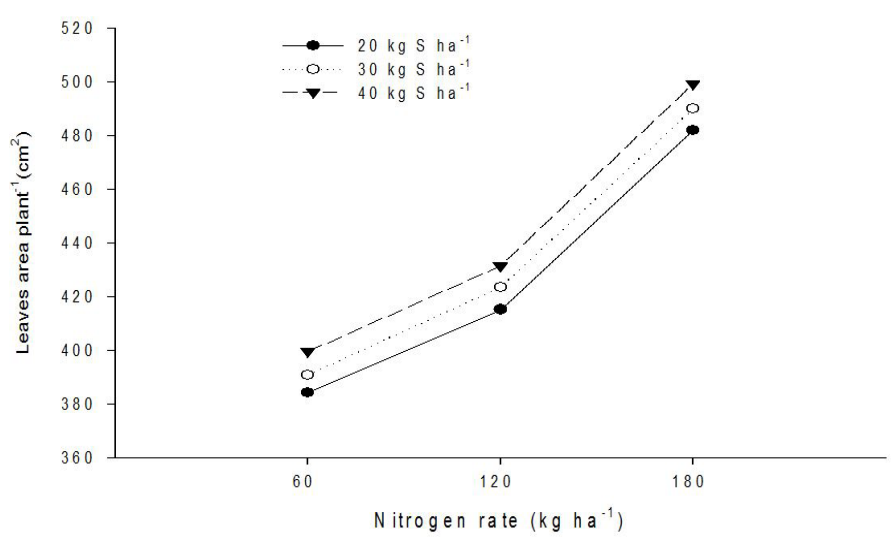

Figure 4: Interaction between $(S x N)$ fertilizers for leaves area plant $^{-1}$ of sesame.

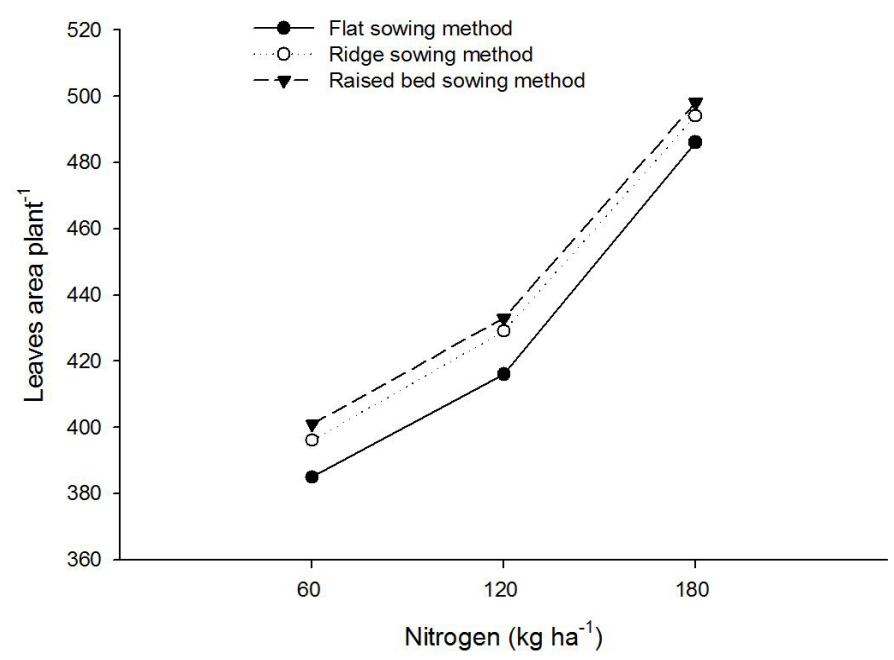

Figure 5: Interaction between nitrogen and sowing methods for leaves area plant ${ }^{-1}$.

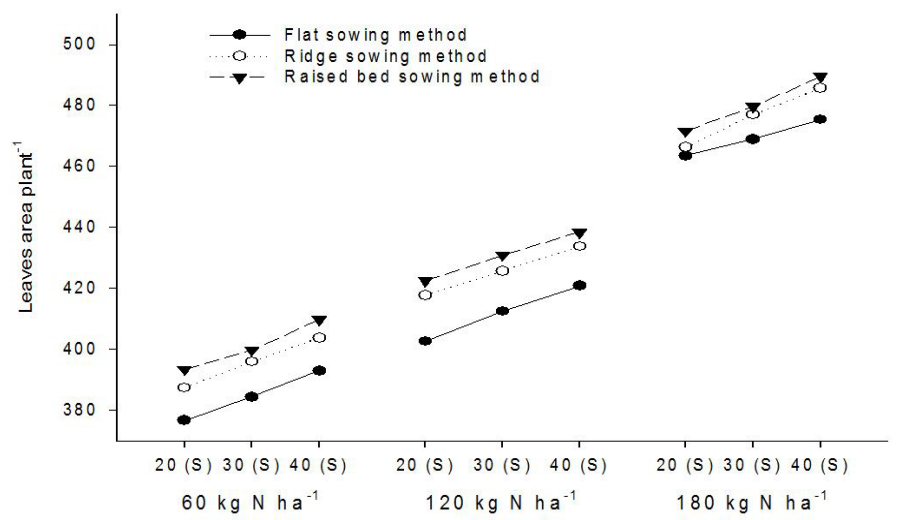

Figure 6: Interaction between sowing methods, nitrogen and sulfur fertilizers leave area plant ${ }^{-1}$ of sesam. 


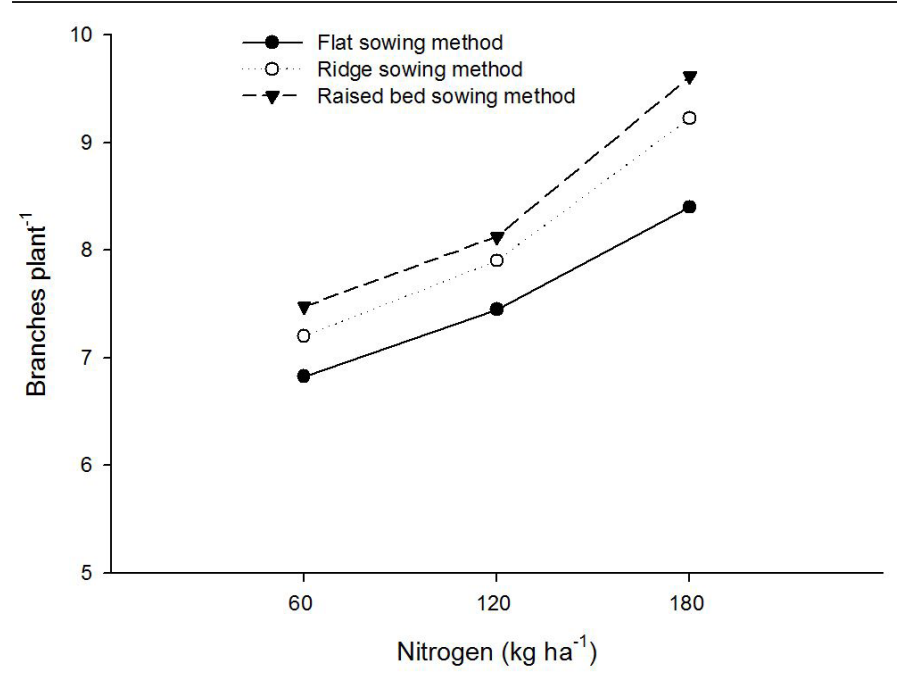

Figure 7: Interaction between $(N x S)$ fertilizers for branches plant ${ }^{-1}$ of sesame.

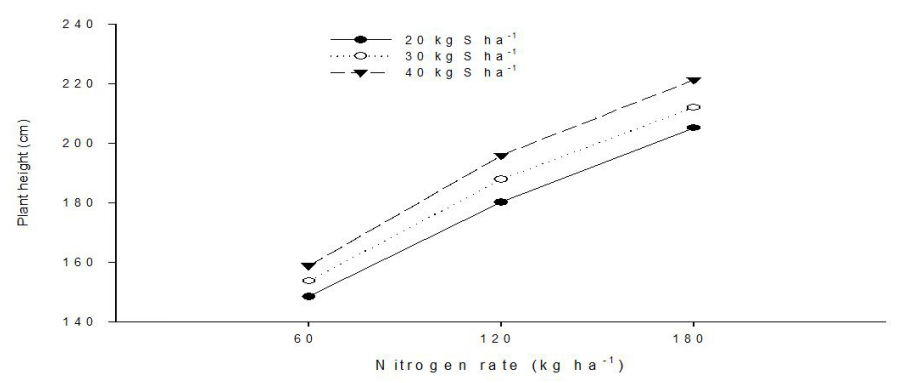

Figure 8: Interaction between nitrogen and sulphur fertilizers for plant height of sesame.

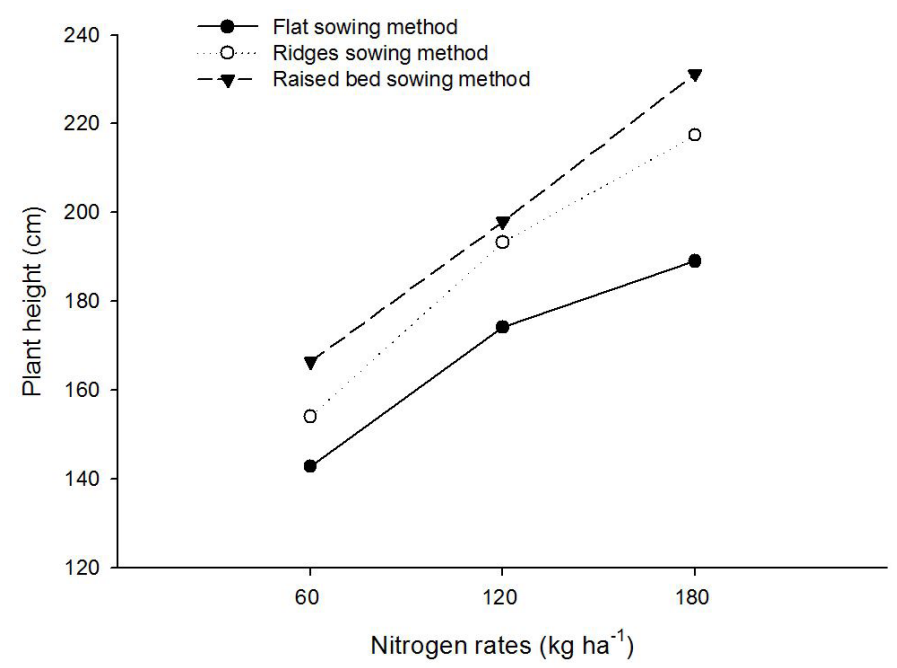

Figure 9: Interaction between nitrogen and sowing methods for plant height of sesame.

aerated soil structure of raised bed, retained more water and nutrients for maximum growth (Haurna, 2011). Interaction between $\mathrm{SM} \times \mathrm{N}$ (Figure 9) shows that branches in raised and ridge sowing method had significantly responded with increase in nitrogen levels.

\section{Plant height $(\mathrm{cm})$}

Table 6, shows data concerning plant height of sesame. Mean comparison values of plant height data showed that different sowing methods, nitrogen and sulfur levels had varied $(p<0.05)$, plant height, whereas combine year variations had significantly changed in sesame plant height. Taller plants were measured from plots sown on raised bed, as compared with other sowing methods, whereas nitrogen and sulfur significantly produced taller plants at $180 \mathrm{~kg} \mathrm{ha}^{-1}$ and $40 \mathrm{~kg} \mathrm{Sha}{ }^{-1}$, respectively. Variation in plant height of raised bed with nitrogen and sulphur application might be due favorable condition for root growth with optimum nutrients utilization in loose and fertile raised bed soil. Purushottam (2005) observed significant increase in plant height with increasing in each increment in nitrogen fertilizer from 50 to 205

Table 5: Branches plant ${ }^{-1}$ of sesame as affected by sowing methods, nitrogen and sulfur levels during 2013 and 2014.

$\begin{array}{llll}\text { Sowing methods } & \mathbf{2 0 1 3} & \mathbf{2 0 1 4} & \text { Mean } \\ \text { Flat } & 8 & 9 & 9 \mathrm{a} \\ \text { Ridge } & 7 & 8 & 8 \mathrm{~b} \\ \text { Raised bed } & 8 & 9 & 9 \mathrm{a} \\ \text { LSD }_{(0.05)} & & & 1\end{array}$

Nitrogen $\left(\mathrm{kg} \mathrm{ha}^{-1}\right)$

$\begin{array}{llll}60 & 7 & 8 & 7 \mathrm{c} \\ 120 & 8 & 9 & 8 \mathrm{~b} \\ 180 & 9 & 10 & 9 \mathrm{a} \\ \text { LSD }_{(0.05)} & & & 1\end{array}$

Sulphur $\left(\mathrm{kg} \mathrm{ha}^{-1}\right)$

$\begin{array}{llll}20 & 8 & 9 & 8 \\ 30 & 9 & 8 & 8 \\ 40 & 8 & 9 & 8\end{array}$

$\begin{array}{llll}\text { LSD }_{(0.05)} & & & \text { ns } \\ \text { Year } & 8 & 9 & *\end{array}$

Planned mean comparison

$\begin{array}{llll}\text { Control } & 5 & 5 & 5 \\ \text { Rest } & 8 & 9 & 8 \\ \text { Significance } & & & *\end{array}$

Interactions

$\begin{array}{lll}\mathrm{Y} \times \mathrm{SM} & \mathrm{ns} & \mathrm{SM} \times \mathrm{S} \quad \mathrm{ns} \\ \mathrm{N} \times \mathrm{S} & \text { ns } & \mathrm{SM} \times \mathrm{N} \times \mathrm{S} \text { ns } \\ \mathrm{Y} \times \mathrm{N} & \text { ns } & \mathrm{Y} \times \mathrm{SM} \times \mathrm{N} \mathrm{ns} \\ \mathrm{Y} \times \mathrm{S} & \text { ns } & \mathrm{Y} \times \mathrm{SM} \times \mathrm{S} n \mathrm{~ns} \\ \mathrm{Y} \times \mathrm{N} \times \mathrm{S} & \text { ns } & \mathrm{Y} \times \mathrm{SM} \times \mathrm{N} \times \mathrm{S} n \mathrm{~s} \\ \mathrm{SM} \times \mathrm{N} & * & \end{array}$

Mean followed different letters are statistically different from each at $5 \%$ of probability; *: indicate significance at $5 \%$ level of probability; ns: indicates not significant. 
Table 6: Plant height (cm) of sesame as affected by sowing methods, nitrogen and sulfur levels during year 2013 and 2014.

\begin{tabular}{|c|c|c|c|}
\hline Sowing methods & 2013 & 2014 & Mean \\
\hline Ridge & 187.89 & 188.50 & $188.19 \mathrm{~b}$ \\
\hline Flat bed & 168.86 & 168.44 & $168.65 \mathrm{c}$ \\
\hline Raised bed & 196.83 & 200.19 & $198.51 \mathrm{a}$ \\
\hline $\mathrm{LSD}_{(0.05)}$ & & & 1.36 \\
\hline \multicolumn{4}{|l|}{ Nitrogen $\left(\mathrm{kg} \mathrm{ha}^{-1}\right)$} \\
\hline 60 & 153.75 & 155.06 & $154.40 \mathrm{c}$ \\
\hline 120 & 187.61 & 189.19 & $188.40 \mathrm{~b}$ \\
\hline 180 & 212.22 & 212.89 & $212.56 \mathrm{a}$ \\
\hline $\operatorname{LSD}_{(0.05)}$ & & & 1.07 \\
\hline \multicolumn{4}{|l|}{ Sulphur (kg ha $\left.{ }^{-1}\right)$} \\
\hline 20 & 177.64 & 178.83 & $178.25 \mathrm{c}$ \\
\hline 30 & 184.33 & 185.39 & $184.86 \mathrm{~b}$ \\
\hline 40 & 191.61 & 192.89 & $192.25 \mathrm{a}$ \\
\hline $\operatorname{LSD}_{(0.05)}$ & & & 1.07 \\
\hline Year & $184.53 \mathrm{~b}$ & $185.71 \mathrm{a}$ & * \\
\hline \multicolumn{4}{|c|}{ Planned mean comparison } \\
\hline Control & 110.33 & 112.00 & 111.17 \\
\hline Rest & 184.53 & 185.71 & 185.12 \\
\hline \multicolumn{4}{|l|}{ Interactions } \\
\hline $\mathrm{Y} \times \mathrm{SM}$ & $*$ & $\mathrm{SM} \times \mathrm{S}$ & \\
\hline $\mathrm{N} \times \mathrm{S}$ & & $\mathrm{SM} \times \mathrm{N} \times \mathrm{S}$ & \\
\hline $\mathrm{Y} \times \mathrm{N}$ & ns & $\mathrm{Y} \times \mathrm{SM} \times \mathrm{N}$ & ns \\
\hline $\mathrm{Y} \times \mathrm{S}$ & ns & $\mathrm{Y} \times \mathrm{SM} \times \mathrm{S}$ & ns \\
\hline $\mathrm{Y} \times \mathrm{N} \times \mathrm{S}$ & $\mathrm{ns}$ & $\mathrm{Y} \times \mathrm{SM} \times \mathrm{N} \times \mathrm{S}$ & ns \\
\hline $\mathrm{SM} \times \mathrm{N}$ & & & \\
\hline
\end{tabular}

Mean followed different letters are statistically different from each at $5 \%$ of probability; *: indicate significance at $5 \%$ level of probability; ns: indicates not significant.

$\mathrm{kg} \mathrm{ha} \mathrm{a}^{-1}$. Nitrogen and sulphur supplied plots resulted in an increase in plant height, while dwarf plants were produced in control plots. Significantly, taller plants $(186 \mathrm{~cm})$ were produced in year 2014, as compared with 2013. Taller Plants were produced with increase in both nitrogen and sulphur levels. Similarly, SM $\times \mathrm{N} \times \mathrm{S}$ interactive response was given in Figure 11 and was found significant for plant height. Plant height in case of flat sowing was lower than the other two sowing methods at each level of nitrogen and sulphur. Interaction between SMxN (Figure 9), and $\mathrm{SMxS}$ (Figure 10) follow same above trend for plant height, the contribution of sulphur negligible as compared with sowing method in Figure 11. The interactive response of nitrogen $(\mathrm{N})$ and sulfur $(\mathrm{S})$ was found significant for plant height (Figure 8). These variations in plant height might be due to good root establishment and development in raised bed with optimum utilization of available nutrients and water. Furthermore, high nitrogen and sulfur doses produce maximum amino acids, assimilates, chloroplast lipids, co-factors, co-enzymes, and photosynthates, which promotes plant height. Haruna (2011) mentioned that plant height increased with synergistic effects of sulfur and nitrogen nutrients in fertile soil.

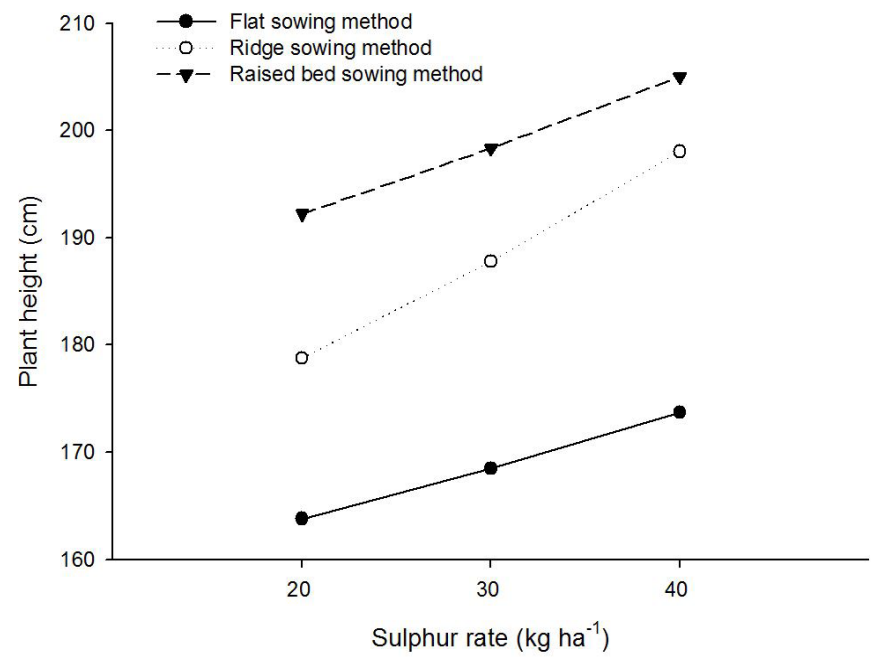

Figure 10: Interaction between sulphur and sowing methods for plant beight of sesame.

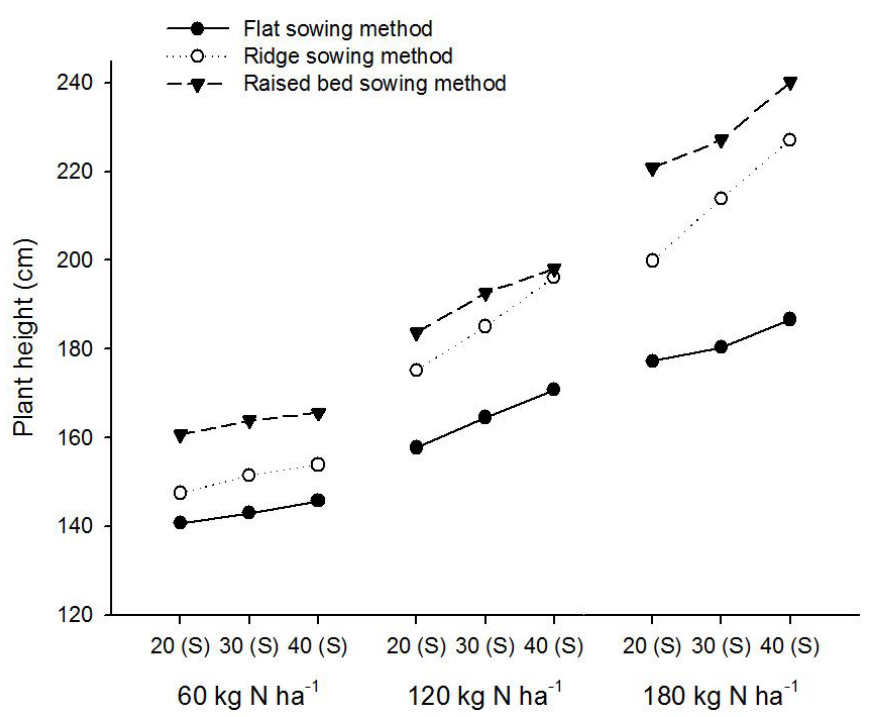

Figure 11: Interaction between sowing methods, nitrogen and sulfur for plant height of sesame.

\section{Seed yield $\left(\mathrm{kg} \mathrm{ha}^{-1}\right)$}

Data exhibited in Table 7, are presenting seed yield and showed that combined two year of seed yield was significantly affected by different sowing methods, different nitrogen and sulfur fertilizer levels, whereas seed yield had significantly changed by year as a 
variant. Higher seed yield $1339 \mathrm{~kg} \mathrm{ha}^{-1}$ was produced in raised bed. Application of nitrogen and sulfur fertilizer at $180 \mathrm{~kg} \mathrm{~N}$ ha ${ }^{-1} 40 \mathrm{Kg} \mathrm{S}$ ha ${ }^{-1}$ produced 1581 and $1346 \mathrm{~kg} \mathrm{ha}^{-1}$ respectively. Maximum seed yield was gained in $2^{\text {nd }}$ year of the experiment as compared with $1^{\text {st }}$ year trail. Tabulated mean data showed that higher seed yield was obtained from fertilized ( $\mathrm{N}$ and S) plots, as compared with no treated plots. The interactive response of $\mathrm{NxS}$ (Figure 12), and $\mathrm{SMxNxS}$ (Figure 13) was found significant for seed yield. Seed yield changes in sesame might be due to the optimum utilization of available nutrients for yield related parameters linked with good soil conditions. It also could be the combine synergistic effect of nitrogen and sulfur on plant growth and development, which

Table 7: Seed yield $\left(\mathrm{kg} \mathrm{ha}^{-1}\right)$ of sesame crop, as affected by sowing methods, nitrogen and sulfur levels during year 2013 and 2014.

\begin{tabular}{|c|c|c|c|}
\hline Sowing methods & 2013 & 2014 & Mean \\
\hline Ridge & 1285 & 1302 & $1294 b$ \\
\hline Flat & 1200 & 1255 & $1228 \mathrm{c}$ \\
\hline Raised bed & 1330 & 1349 & $1339 \mathrm{a}$ \\
\hline $\mathrm{LSD}_{(0.05)}$ & & & 34 \\
\hline \multicolumn{4}{|l|}{ Nitrogen $\left(\mathrm{kg} \mathrm{ha}^{-1}\right)$} \\
\hline 60 & 988 & 986 & $987 \mathrm{c}$ \\
\hline 120 & 1288 & 1298 & $1293 \mathrm{~b}$ \\
\hline 180 & 1540 & 1621 & $1581 \mathrm{a}$ \\
\hline $\mathrm{LSD}_{(0.05)}$ & & & 21 \\
\hline \multicolumn{4}{|l|}{ Sulphur $\left(\mathrm{kg} \mathrm{ha}^{-1}\right)$} \\
\hline 20 & 1208 & 1256 & $1232 \mathrm{c}$ \\
\hline 30 & 1264 & 1302 & $1283 \mathrm{~b}$ \\
\hline 40 & 1344 & 1348 & $1346 \mathrm{a}$ \\
\hline $\mathrm{LSD}_{(0.05)}$ & & & 21 \\
\hline Year & 1272 & 1302 & * \\
\hline \multicolumn{4}{|c|}{ Planned mean comparison } \\
\hline Control & 601 & 661 & 631 \\
\hline Rest & 1272 & 1302 & 1287 \\
\hline Significance & & & $*$ \\
\hline \multicolumn{4}{|l|}{ Interactions } \\
\hline $\mathrm{Y} \times \mathrm{SM}$ & ns & $\mathrm{SM} \times \mathrm{S}$ & ns \\
\hline $\mathrm{N} \times \mathrm{S}$ & $*$ & $\mathrm{SM} \times \mathrm{N} \times \mathrm{S}$ & \\
\hline $\mathrm{Y} \times \mathrm{N}$ & $*$ & $\mathrm{Y} \times \mathrm{SM} \times \mathrm{N}$ & ns \\
\hline $\mathrm{Y} \times \mathrm{S}$ & ns & $\mathrm{Y} \times \mathrm{SM} \times \mathrm{S}$ & ns \\
\hline $\mathrm{Y} \times \mathrm{N} \times \mathrm{S}$ & ns & $\mathrm{Y} \times \mathrm{SM} \times \mathrm{N} \times \mathrm{S}$ & ns \\
\hline $\mathrm{SM} \times \mathrm{N}$ & ns & & \\
\hline
\end{tabular}

Mean followed different letters are statistically different from each at $5 \%$ of probability; ***: indicate significance at $5 \%$ level of probability; ns: indicates not significant.

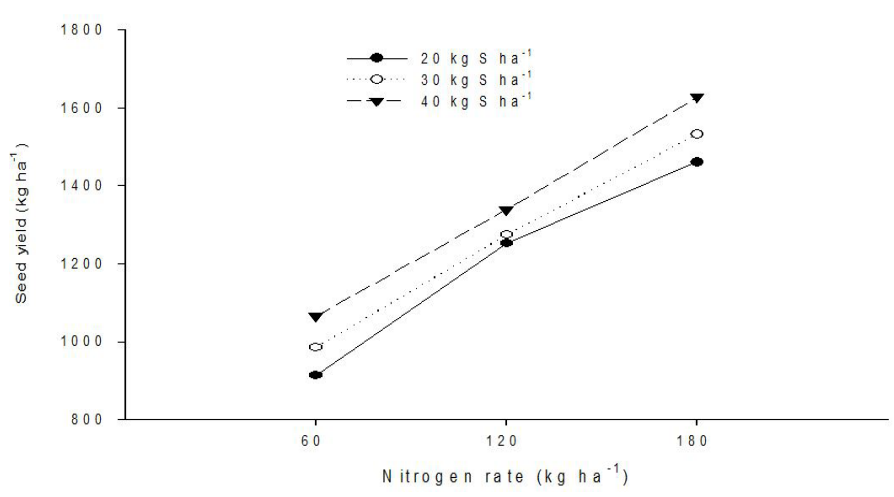

Figure 12: Interaction between nitrogen and sulfur fertilizers for seed yield of sesame.

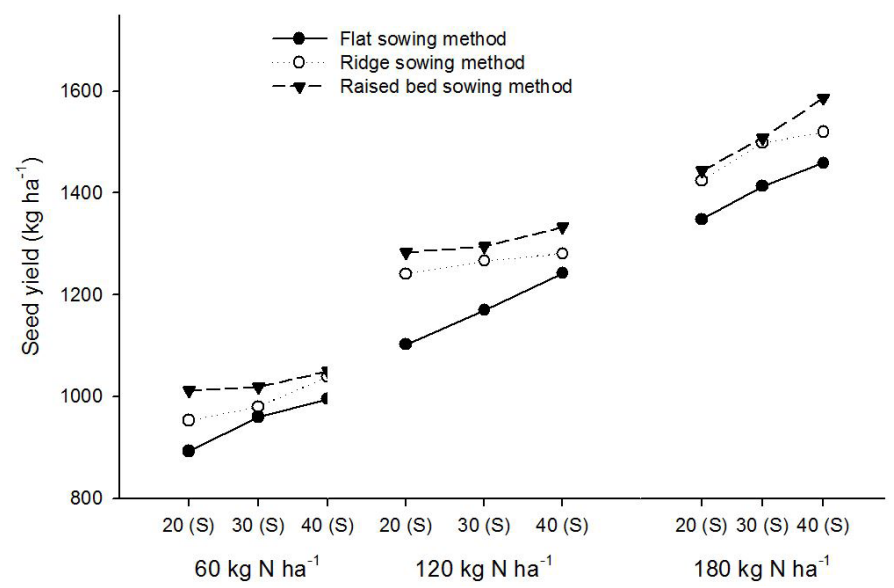

Figure 13: Interaction sowing methods, between nitrogen and sulfur fertilizers for sesame seed yield.

enhance branches, seed, capsule plant ${ }^{-1}$, plant height and resulted in maximum seed production. Good soil condition with optimum $\mathrm{N}$ and $\mathrm{S}$ nutrients availability encouraged overall growth and development process of crops.

\section{Conclusions and Recommendations}

Sesame has tremendously responded to raised bed sowing method. Sowing of sesame on raised bed followed by ridge had significantly improved leaf, stem and yield parameters. Combined application of nitrogen at $180 \mathrm{~kg} \mathrm{ha} \mathrm{h}^{-1}$ and sulphur $40 \mathrm{~kg} \mathrm{ha}^{-1} \mathrm{had}$ promoted yield related parameters and yield. The combined application of $\mathrm{N}$ and $\mathrm{S}$ at $180 \mathrm{~kg} \mathrm{ha}^{-1}$ and $40 \mathrm{~kg} \mathrm{ha}{ }^{-1}$ in raised seed bed is recommended for achieving higher sesame yield production.

\section{Novelty Statement}

Novelty of this research is an adaptation of standard agronomic land management and nutrients management techniques, which can improve the productivity of sesame crops. Further, it has been revealed that ses- 
ame sown on well aerated loose soil of raised bed and ridge bed with combination of different nitrogen and sulphur fertilizers had improved yield and yield related parameters of the sesame.

\section{Author's Contribution}

Alam Zeb: Principal author, who conducted research, field experiments and analysis. Further, wrote draft of the manuscript.

Amanullah Jan: Major Supervisor, who supervised whole research study and provided technical guidelines.

\section{Conflict of interest}

The authors have declared no conflict of interest.

\section{References}

Abad, M.B., M.D. Clement, R.P. Aragan and A.S. Camarero. 1997. The influence of soil urban waste compost and nitrogen mineral fertilizer on growth and productivity in potatoes. Commun. Soil Sci. Plant Anal., 28(17-18): 1653-1661. https://doi.org/10.1080/00103629709369905

Abdel-Rahman, A. and E. Mahdi. 2008. Response of sesame to nitrogen and phosphorus fertilization in Northern Sudan. J. Appl. Biosci., 8(2): 303-308.

Ahmad, A. and M.Z.Abdin.2000. Interactive effect of Nitrogen and Sulfur on the oil and protein Contents and on the fatty acid profiles of oil in the seeds of rapeseed (Brassica campestris L.) and Mustard (Brassica juncea L. Czern. and Coss.). J. Agro. Crop Sci., 185(1): 49-54. https://doi. org/10.1046/j.1439-037X.2000.00401.x

Ahmad, A., M. Akhtar, A. Hussain, Ehsanullah and M. Musaddique. 2001. Genotypic response of sesame to nitrogen and phosphorus application. Pak. J. Agri. Sci., 38(2): 12-15.

Ashri, A., 2010. Sesame breeding. In plant breeding reviews. Janick J. Oxford: John Wiley. (16). http:// faostat.fao.org/site/567/default, https:// doi.org/10.1002/9780470650110.ch5

Asl, A.N. 2017. Effects of nitrogen and phosphate biofertilizers on morphological and agronomic characteristics of sesame (Sesamum indicum L.). Open J. Ecology. 7: 101- 111.

Bareja B.G., 2011. Topography and soil, abiotic factors affecting crop growth, development and productivity. Crops Review. https://www. cropsreview.com/abiotic-factors.html

Bremer, J.M and C.S. Mulveni. 1982. Nitrogen. In: A.L. Page, R.H. Miller and D.R. Keeney (eds.). Methods of soil analysis. Part-2. USDA. pp. 595-682.

Cheung, S.C., Y.T. Szeto and I.F. Benzie. 2007. Antioxidant protection of edible oils. Plant Foods Hum. Nutri. 62(1): 39-42.

Daremcar Annual Report, 2006-2007. Agricultural engineering and technology. Agric. Sci. Univ. of California, USA. pp. 251.

Deshmukh, V.A., D.A. Chavan and G.T. Sugave. 1990. Response of summer sesame (Sesamum indicum L.) to nitrogen and phosphorus. Ind. J. Agron., 37(3): 604-605.

EI-Nakhlawy, F. and M.A. Shaheen. 2009. Response of seed yield, yield components and oil content of the sesame cultivar and nitrogen fertilizer rate diversity. JKAU: Met., Environ. Arid Land Agric. Sci., 20(2): 21-31. https://doi. org/10.4197/met.20-2.2

El-Serogy, S.T., M.A. El-Eman and W.A.I. Sorour. 1997. The performance of two sesame varieties under different sowing method in two locations. Ann. Agric. Sci., 42: 355-364.

FAO, 2014. Report on sulphur in agriculture. Fert. News. 49:1-5.http://www.fao.org/publications/ sofa/2014/en/

Food and Agriculture Organization of the United Nations. 2012. Production crops: Sesame seeds. http://www.fao.org/publications/sofa/2012/en/

Ghosh, D.C. and J.C. Sen. 1980. Analysis of yield components of sesamum as influenced by nitrogen and phosphorus fertilization. Madras Agric. J., 67(1): 9-13.

Ghosh, D.C., and M. Maji. 1985. Production efficiency and economics of $\mathrm{N}$ and $\mathrm{P}$ fertilization to sesame (sesamum indicum L.) under lateritic acid soil. Oilseeds J., 13: 44-49.

Haruna, I.M., 2011. Dry matter partitioning and grain yield potential in sesame (Sesamum indicum L.) as influenced by poultry manure, nitrogen and phosphorus at Samaru, Nigeria. J. Agric. Tech., 7(6): 1571-1577. www.ijat-aatsea. com

Hasanpour, R., H. Pirdashti, M.A. Esmaeili and A. Abbasian. 2012. Effect of plant growth promoting rhizobacterial (PGPR) and nitrogen on qualitative characteristics of sesame (Sesamum indicum L.) cultivars. Inter. J. Agri. Crop Sci., 4(11): 662-665. 
Hossein, M.A., A. Hamid and S. Nasreen 2007. Effect of nitrogen and phosphorus fertilizer and $\mathrm{P}$ uptake and yield performance of sesame. J. Agric. Res., 45(2): 119-127.

Jadav, D.P., D.R. Padamani, K.B. Polara, K.B. Parmar and N.B. Babaria 2010. Effect of different level of sulphur and potassium on growth, yield and yield attributes of sesame (Sesamum Indicum L.). Asian J. Soil. Sci., 5(1): 106-108. https://www.semanticscholar.org

Jain, H.C.,M.R. Deshmukh,U. Goswami and D.M. Hedge. 2000. Studies on land configuration and seed hardening on productivity of sesame in different soil types. J. Maharastra Agric. Univ., 25(1): 1-2. https://www.cabdirect.org/ cabdirect/abstract/20013031789

Jain, H.C., M.R. Deshmukh, U. Goswami and D.M. Hedge.1999. Studies on time and method of sowing under rainfed conditions in vertisols for sesame production. J. Maharastra Agric. Univ., 24(2): 181-183.

Jamal, A., K. Ko, H.S. Kim, Y.K. Cho, H. Joung and K. Ko. 2009. Role of genetic factors and environmental conditions in recombinant protein production for plant molecular biofarming. Biotech. Adv., 27: 914-923. https:// doi.org/10.1016/j.biotechadv.2009.07.004

Jan, M.T., P. Shah, P.A. Hoolinton, M.J. Khan and Q. Sohail. 2009. Agriculture research: Design and analysis. Dept. Agron. Univ. Agric. Peshawar, Pakistan. https://scholar.google.com/ citations? user $=$ pfZbB04AAAAJandhl=en

Jouyban, Z. and S.G. Moosave. 2011. Study of effects of different levels of irrigation interval, nitrogen and super absorbent on seed yield and morphological traits of sesame. Aust. J. Basic Appl. Sci., 5(10): 1317-1323.

Jouyban, Z. and S.G. Moosavi. 2011. Effect of different irrigation intervals, nitrogen and super absorbent levels on chlorophyll index, yield and yield components of sesame. Islamic Azad University, Birjand, Jonuby Khorasan province. Iran. J. Food Agric. Environ., 10(1): 360-364.

Kamal-Eldin, A., M. Ali and W. Sidiga. 2011. Sesame seed lignans: Potent physiological modulators and possible ingredients in functional foods and nutraceuticals. Recent Patents Food, Nutri. Agric. 3: 17-29.

Langham, D.R., 2008. Growth and development of sesame. American Sesame Growers Association. https://www.researchgate.net/publication/
265308920 _growth_ and_development of sesame

Leustek, T. and K. Saito. 1999. Sulfate transport and assimilation in plants. Plant Physiol., 120: 637-643.https://doi.org/10.1104/pp.120.3.637

Mahale, M.M., V.B. Nevase and P.G. Chavan. 2008. Yield of sesame (Sesamum indicum L.) and groundnut (arachishypogaea) as influenced by different intercropping ratios and sulphur levels. Dapoli Dist. Ratnagiri, India. Legume Res., 31(4): 268-271.

Malik, A.M., M.F. Saleem, M.A. Cheema and S. Ahmed. 2003. Influence of different nitrogen levels on productivity of sesame (Sesamum indicum L.) under varying planting patterns. Int. J. Agri. Bio., 5(4): 490-492.

Mansoori, I. 2012. Response of canola to nitrogen and sulphur fertilizer. Int. J. Agri. Crop Sci., 4(1): 28-33.

McGrath, S.P. and F.J. Zhao. 1996. Sulphur uptake, yield response and the interactions between $\mathrm{N}$ and $\mathrm{S}$ in winter oilseed rape (Brassica napus L.) J. Agric. Sci., 126: 53-62. https://doi. org/10.1017/S0021859600088808

MINFA, 2015-16. Ministry for food and agriculture, agriculture statistics of Pakistan. Govt. of Pak. Economic Wing, Islamabad. pp. 33. http://www.mnfsr.gov.pk/pubDetails.aspx

Mondal, M., M. Badruddin, M. Malek, M. Hossainand A. Puteh. 2012. Optimization of sulphur requirement to sesame (Sesamum indicum L.) genotypes using tracer techniques. Bangladesh J. Bot., 41(1): 7-13. https://doi. org/10.3329/bjb.v41i1.11076

Purushottam, G. 2005. Integrated nutrient management in sesame (Sesamum indicum L.) and its residual effect in succeeding chickpea (Cicer arietinum L.). M.Sc thesis, Uni. of Agri. Sci., Dharwad, India.

Radford, P.J., $1967 . \quad$ Growth Analysis formula, their uses and abuses. Crop Sci., 3: 171-173. https://doi.org/10.2135/ cropsci1967.0011183X000700030001x

Raja, A., K.O. Hattab, L. Gurusamy and S. Suganya. 2007. Suphur levels on nutrients uptake and yield of sesame varieties and nutrients availability. Tamil Nadu Agricultural University, Coimbatore, India. Int. J. Soil Sci., 2(4): 278-285.

Ramakrishnan, A., A. Sundaram and K. Appavoo. 1994. Influence of fertilization on yield and 
components of sesame (Sesamum indicum L.). Madras. Agric. J., 81: 696-698.

Ray, D.L., S. Glenn, W. Terry and W. Mark. 2008. Southwest sesame growers guide. https://baylor.agrilife.org/files/2011/05/ sesamegrowerguide2008.pdf

Roy, S.K., S.M.L. Rahaman and A.B.M. Salahudin. 1995. Effect of nitrogen and potassium on growth and seed yield of sesame (Sesamum indicum L). Indian J. Agric. Sci., 65(7): 509-511.

Scherer, H.W., 2001. Sulphur in crop production invited paper. Eur.J.Agron., 14: 81-111.https:// doi.org/10.1016/S1161-0301(00)00082-4

SESACO, Sesame Coordinators. 2014. Sesame Producer Guide. pp. 1- 6.

Shilphi, S., M. Nuruzzaman, F. Akhter, M.N.
Islam and G.N.C. Sutradher. 2014. Response of nitrogen and sulfur on the oil content of sesame and nutrient status of soil. Int. J. Bioresour. Stress Manage., 5(1): 41-46. https://doi. org/10.5958/j.0976-4038.5.1.007

Toan, D.P., T.N.A. Thuy-Duong, S. Carlsson and T.M. Bui. 2010. Morphological evaluation of sesame (Sesamum indicum L.) varieties from different origins. A. J. Crop. Sci., 4(7): 498-504.

Younis, M., S. Shah, Inamullah, R. Gul, A. Jalal, F. Khalil, I. Hussain and M.A. Fahad.2020. Effect of phosphorus and sulphur on yield and yield components of sesame. Sarhad J. Agric., 36(2): 722-728. http://dx.doi.org/10.17582/journal. sja/2020/36.2.722.728 